\title{
V363 Cassiopeiae: a new lithium-rich Galactic Cepheid ${ }^{\star}$
}

\author{
G. Catanzaro ${ }^{1}$, V. Ripepi ${ }^{2}$, G. Clementini ${ }^{3}$, F. Cusano ${ }^{2}$, G. De Somma ${ }^{2}$, S. Leccia ${ }^{2}$, M. Marconi², R. Molinaro², \\ M. I. Moretti ${ }^{2}$, I. Musella ${ }^{2}$, and V. Testa ${ }^{4}$ \\ 1 INAF-Osservatorio Astrofisico di Catania, Via S.Sofia 78, 95123 Catania, Italy \\ e-mail: giovanni.catanzaro@inaf.it \\ 2 INAF-Osservatorio Astronomico di Capodimonte, Salita Moiariello 16, 80131 Naples, Italy \\ e-mail: vincenzo.ripepi@inaf.it \\ 3 INAF-Osservatorio di Astrofisica e Scienza dello Spazio, Via Gobetti 93/3, 40129 Bologna, Italy \\ 4 INAF - Osservatorio Astronomico di Roma, via Frascati 33, 00078 Monte Porzio Catone, Italy
}

Received 25 May 2020 / Accepted 4 June 2020

\begin{abstract}
Context. Classical Cepheids (DCEPs) are important astrophysical objects not only as standard candles in the determination of the cosmic distance ladder, but also as a testbed for the stellar evolution theory. This is based on the strict connection between their pulsation (period(s) and amplitudes) and stellar parameters (luminosity, mass, effective temperature, and metallicity).

Aims. We examine the nature of the Galactic DCEP V363 Cas and other DCEPs that show cosmic abundances of lithium in their atmospheres.

Methods. We collected three epochs of high-resolution spectroscopy for V363 Cas with HARPS-N at the TNG. We measured accurate stellar parameters: the effective temperatures, gravities, microturbulences, radial velocities, and metal abundances.

Results. We detected a lithium abundance of $\mathrm{A}(\mathrm{Li})=2.86 \pm 0.10 \mathrm{dex}$, along with iron, carbon, and oxygen abundances of $[\mathrm{Fe} / \mathrm{H}]=-0.30 \pm 0.12 \mathrm{dex},[\mathrm{C} / \mathrm{H}]=-0.06 \pm 0.15 \mathrm{dex}$, and $[\mathrm{O} / \mathrm{H}]=0.00 \pm 0.12 \mathrm{dex}$. V363 Cas is the fifth of the Milky Way DCEPs to exhibit a Li-rich feature. An analysis of historical time-series spanning a 100-year interval shows that the period of V363 Cas is increasing, with a sharp acceleration after HJD $=2453000$ days. This is a clear indication of a first crossing of the instability strip. Conclusions. Our results favour the scenario in which the five Galactic Li-rich DCEPs are on their first crossing of the instability strip and have had slowly rotating progenitors during their main-sequence phase.
\end{abstract}

Key words. stars: variables: Cepheids - pulsars: individual: V363 Cas - stars: abundances - stars: fundamental parameters Hertzsprung-Russell and C-M diagrams - stars: evolution

\section{Introduction}

Classical Cepheids (DCEPs) are the primary Population I standard candle within the Local Group. The DCEPs with the shortest periods are likely associated with the first crossing of the instability strip (IS) and are expected to show peculiar chemical features compared with the canonical, more evolved blue loop pulsators.

In this Letter we report the discovery of a new rare lithiumrich DCEP. Only four DCEPs showing an enhanced lithium abundance (through detection of the Li I $6707.766 \AA$ ) have been discovered in the Galaxy so far (Luck \& Lambert 2011; Kovtyukh et al. 2016, 2019), and an additional DCEP was detected in the Large Magellanic Cloud (LMC, Luck \& Lambert 1992). All these objects show a lithium abundance $\mathrm{A}(\mathrm{Li}) \sim 3.0$ dex, while the majority of the Galactic DCEPs show $\mathrm{A}(\mathrm{Li})<1.2$ dex (Luck \& Lambert 2011). This discovery was surprising as $\mathrm{Li}$ is expected to be depleted by proton-capture after the first dredge-up (1DU) that occurs at the beginning of the red giant branch (RGB) phase (Iben 1967). A natural explanation is that these DCEPs are at their first crossing of the IS and their

\footnotetext{
^ Based on observations made with the Italian Telescopio Nazionale Galileo (TNG) operated by the Fundación Galileo Galilei (FGG) of the Istituto Nazionale di Astrofisica (INAF) at the Observatorio del Roque de los Muchachos (La Palma, Canary Islands, Spain).
}

envelopes do not show the signature of nuclear processes that occurred during the main-sequence (MS) phase. According to Kovtyukh et al. (2019), at least three of out of the four Milky Way (MW) Li-rich DCEPs also show abundances of the CNO species that are consistent with the solar values, that is, they are not processed by the $\mathrm{CN}$-cycle. However, the 1DU is not the only phenomenon capable of depleting lithium. Rotational mixing can reduce the lithium abundance by a factor of one hundred in a fraction of the MS lifetime, for sufficiently fast rotating MS stars (e.g. Brott et al. 2011). This would then explain the low number of Li-rich DCEPs. As noted by Kovtyukh et al. (2019), about $80 \%$ of the DCEPs that are expected to be at their first crossing (about $5 \%$ of the total) are Li-depleted. Therefore it can be hypothesised that the progenitors of the Li-rich and Li-depleted DCEPs (B stars) were slow and fast rotating stars, respectively, when they were on the MS. It is known that a fraction $(\sim 15 \%)$ of the B-stars show $v \sin i<20 \mathrm{~km} \mathrm{~s}^{-1}$ (Huang et al. 2010), while the large majority rotates much faster. It is thought that the slow rotators loose most of their angular momentum on the MS due to stellar winds that are enhanced by the rotation itself (Maeder \& Meynet 2000). When they become DCEPs, they therefore show the moderate rotational velocities typical of these stars.

An additional feature of the Li-rich DCEPs is that they most frequently are multi-mode pulsators. Of the four MW 
Li-rich DCEPs, ASAS J075842-2536.1 and ASAS J1317146605.0 both pulsate in the first and second overtone (DCEP_1O2O), V371 Per pulsates in the fundamental and first overtone (DCEP_F1O), and V1033 Cyg is only a fundamental mode (DCEP_F) pulsator. According to Kovtyukh et al. (2019), multi-mode DCEPs have a less efficient mixing in their envelope than DCEP_F, which means that they would preferentially tend to retain their $\mathrm{Li}$.

Even if other more complex processes can address the presence of lithium in DCEPs (see Kovtyukh et al. 2019, for a detailed discussion), the basic mechanism to explain Li-rich DCEPs is their passage through the IS at the first crossing. This occurrence can be verified by measuring the rate of period change that is due to evolution along the Hertzsprung-Russell diagram (HRD), as the period is expected to increase at the first and third crossing but to decrease at the second crossing (see e.g. Turner et al. 2006). The data available to date allowed Kovtyukh et al. (2019) to detect a quick period change in V1033 Cyg, but they were insufficient to detect period changes in the other three MW DCEPs.

In the course of a large project devoted to measuring the chemical abundance of 100 un-characterised or newly discovered Galactic DCEPs, we obtained high-resolution spectroscopy for V363 Cas and discovered a deep Li I $6707.766 \AA$ line in the spectra of this MW DCEP. V363 Cas has long been considered a fundamental mode (ab-type) RR Lyrae variable with a period $P \sim 0.546$ days (e.g. Nowakowski 1988). However, Hajdu et al. (2009) showed that the star is a multi-mode DCEP pulsating in the first (1O) and second (2O) overtone modes, with a period ratio of $\mathrm{P} 2 / \mathrm{P} 1 \sim 0.801$, which is typical of such multi-mode DCEPs (see e.g. Fig. 3 in Udalski et al. 2018). More recently, Prudil et al. (2020) noticed that V363 Cas is not a RR Lyrae star on the basis of its Gaia parallax. Nevertheless, a number of recent papers still erroneously considered V363 Cas as an RR Lyrae star (e.g. Dambis et al. 2013). Furthermore, Kervella et al. (2019a,b) claimed that V363 Cas is a binary RR Lyrae with a low-mass companion that has an upper limit of $0.2 M_{\odot}$.

In the following we confirm the Hajdu et al. (2009) classification of V363 Cas as a multi-mode DCEP. We base this on the position of this star on the HRD and on an analysis of the photometric time-series data.

\section{Spectroscopic observations and data analysis}

Multi-phase spectroscopic observations of V363Cas were obtained at the $3.5 \mathrm{~m}$ Telescopio Nazionale Galileo (TNG), which is equipped with the HARPS-N instrument, in three nights on November 21, 26, and December 19, 2019. HARPS-N features an echelle spectrograph covering the wavelength range between 3830 to $6930 \AA$, with a spectral resolution $R=115000$. The signal-to-noise ratio $(\mathrm{S} / \mathrm{N})$ varies from 50 to 100 at $\lambda=$ $5000 \AA$. The main characteristics of V363 Cas are summarised in Table 1.

Reduction of all the spectra, which included bias subtraction, spectrum extraction, flat fielding, and wavelength calibration, was performed using the HARPS reduction pipeline. Radial velocities were measured by cross-correlating each spectrum with a synthetic template, using the IRAF task FXCOR and excluding Balmer lines as well as wavelength ranges containing telluric lines. The IRAF package RVCORRECT was adopted to determine the heliocentric velocity by correcting the spectra for the Earth's motion.

To measure the elemental abundances, we first need to estimate main stellar atmospheric parameters such as the effective
Table 1. Main characteristics of V363 Cas.

\begin{tabular}{ccccc}
\hline \hline Gaia ID & $l$ & $b$ & $\langle V\rangle$ & $\begin{array}{c}E(B-V) \\
\text { mag }\end{array}$ \\
\hline 429162271910068352 & 118.46 & -02.217 & 10.550 & $0.437^{(a)}$ \\
\hline
\end{tabular}

Notes. $\langle V\rangle$ is the intensity-averaged magnitude of the star. References. ${ }^{(a)}$ Kervella et al. (2019b).

temperature $\left(T_{\text {eff }}\right)$, surface gravity $(\log g)$, microturbulence $(\xi)$ and the total line broadening, which is measured in our spectra equal to $15 \pm 1 \mathrm{~km} \mathrm{~s}^{-1}$. A $T_{\text {eff }}$ value for each spectrum of V363 Cas was estimated using the line depth ratio (LDR) method (Kovtyukh \& Gorlova 2000), which is commonly used in the literature for DCEPs. We measured about 32 LDRs in each spectrum. An iterative procedure was then applied to determine the microturbulent velocity $\xi$, iron abundance $[\mathrm{A}(\mathrm{Fe})]$, and $\log g . \xi$ values were estimated by demanding the slope of the iron abundance as a function of the equivalent width (EW) to be null, that is, the iron abundance to not depend on EWs. To this purpose, we used a sample of 145 Fe I spectral lines, extracted from the line list of Romaniello et al. (2008). The iron content was estimated by converting the measured EWs into abundances through the WIDTH9 code (Kurucz \& Avrett 1981) after generating an appropriate model atmosphere with the ATLAS9 LTE code (Kurucz 1993a,b). At this stage, we neglected the $\log g$ because the $\mathrm{Fe}$ I lines are insensitive to this parameter. EWs were measured using an IDL $^{1}$ semi-automatic custom routine, which allowed us to minimise errors in the continuum evaluation on the wings of the spectral lines. Then, we estimated the surface gravity by imposing the ionisation balance between Fe I and Fe II lines. For Fe II, we used a list of 24 lines extracted from the compilation by Romaniello et al. (2008). The atmospheric parameters derived for each spectrum of V363 Cas are summarised in Table 2. Because we obtained consistent temperatures and gravities for each night, we adopted the weighted average values, that is, $T_{\text {eff }}=6660 \pm 100 \mathrm{~K}$ and $\log g=2.0 \pm 0.1 \mathrm{dex}$, to reproduce the observed spectral energy distribution (SED) with the synthetic flux computed using the ATLAS 9 code. The observed flux was retrived from the VOSA tool (Bayo et al. 2008) and corrected for reddening by adopting $E(B-V)=0.437 \mathrm{mag}$ (Kervella et al. 2019b) and the Fitzpatrick (1999) extinction law. The comparison between observed and the theoretical SEDs is shown in Fig. 1. Furthermore, using the distance inferred from the Gaia DR2 parallax $(\pi=0.7669 \pm 0.0278$ mas, to which we applied a zero-point correction of 0.049 mas; Groenewegen 2018), we derived a bolometric luminosity of $L / L_{\odot}=260 \pm 49$ for V363 Cas.

The atmospheric parameters listed in Table 2 were used as input for the abundance analysis, which was performed following the procedures in Catanzaro et al. (2019). For the analysis, we used the spectrum acquired on November 26, 2019, which has the highest $\mathrm{S} / \mathrm{N}$ (up to 100). The abundances of the 26 species we detected in this spectrum are provided in Table 3. In Fig. 2 we show the comparison between observed and synthetic spectra in four main spectral regions: $\mathrm{H} \beta$ and $\mathrm{H} \alpha$ (to confirm the effective temperature), the $\mathrm{Mg}$ I triplet (to confirm $\log g$ ), and the Li I $6708.766 \AA$ line. For the synthesis of the Li I line, we took the hyperfine structure and the close Fe I $6708.282 \AA$ line into account.

1 IDL (Interactive Data Language) is a registered trademark of Harris Geospatial Solutions. 
Table 2. Atmospheric parameters.

\begin{tabular}{cccccccc}
\hline $\begin{array}{c}\text { HJD } \\
2400000+\end{array}$ & Phase & $\begin{array}{c}T_{\text {eff }} \\
(\mathrm{K})\end{array}$ & $\begin{array}{c}\log g \\
(\mathrm{dex})\end{array}$ & $\begin{array}{c}\xi \\
\left(\mathrm{km} \mathrm{s}^{-1}\right)\end{array}$ & $\begin{array}{c}v_{\mathrm{rad}} \\
\left(\mathrm{km} \mathrm{s}^{-1}\right)\end{array}$ & $\mathrm{A}(\mathrm{Fe})$ & $\mathrm{A}(\mathrm{Li})$ \\
\hline 58809.5196 & 0.996 & $6650 \pm 170$ & $1.9 \pm 0.2$ & $2.1 \pm 0.6$ & $-55.2 \pm 0.1$ & $7.24 \pm 0.12$ & $2.86 \pm 0.10$ \\
58814.4665 & 0.046 & $6620 \pm 180$ & $2.0 \pm 0.2$ & $2.4 \pm 0.6$ & $-55.9 \pm 0.1$ & $7.19 \pm 0.12$ & $2.86 \pm 0.10$ \\
58837.3996 & 0.002 & $6710 \pm 160$ & $2.2 \pm 0.3$ & $2.1 \pm 0.5$ & $-50.2 \pm 0.1$ & $7.28 \pm 0.12$ & $2.86 \pm 0.10$ \\
\hline
\end{tabular}

Notes. For each spectrum of V363 Cas we list the heliocentric Julian date (HJD) at mid exposure (Col. 1), pulsation phase (Col. 2), effective temperature (Col. 3), gravity (Col. 4), microturbulent and radial velocities (Cols. 5 and 6), iron and lithium abundances (Cols. 7 and 8) expressed in a logarithmic scale relative to hydrogen.

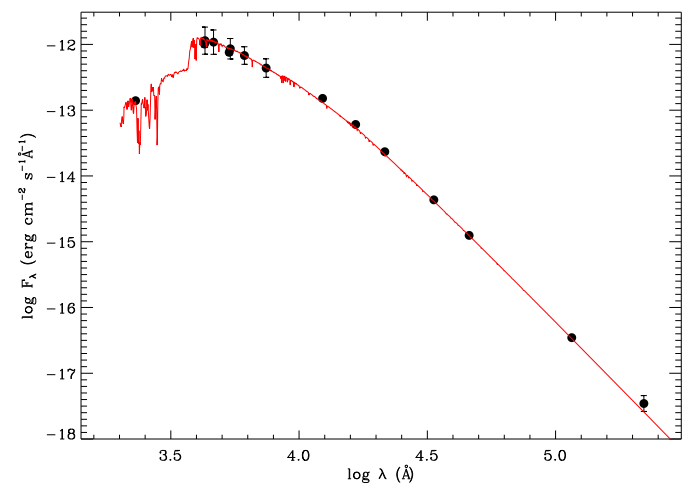

Fig. 1. Spectral energy distribution of V363 Cas. Filled dots represent the observed fluxes as retrieved from the VOSA tool. The red line shows the theoretical flux computed using the ATLAS9 model for $T_{\text {eff }}=6660 \mathrm{~K}$ and $\log g=2.0 \mathrm{dex}$.

As a general trend, V363 Cas appears to be slightly metal poor because the iron peak elements are under-abundant with respect to the solar composition (Grevesse et al. 2010). The low iron content, $[\mathrm{Fe} / \mathrm{H}] \approx-0.30 \mathrm{dex}$, is consistent with a previous estimate by Fernley \& Barnes (1997) ( -0.38 dex). We paid particular attention to light elements involved in the 1DU mixing, such as carbon, oxygen, and sodium ${ }^{2}$. The carbon and oxygen abundances agree with the solar values, while sodium is slightly under-abundant. The lithium line was reproduced with an abundance of $\mathrm{A}(\mathrm{Li})=2.86 \pm 0.10 \mathrm{dex}$. This value agrees well with results from the standard Big Bang nucleosynthesis theory, which predicts a lithium abundance of $\mathrm{A}(\mathrm{Li})=2.72 \pm 0.06 \mathrm{dex}$ (Cyburt et al. 2008).

\section{Time-series analysis and period change}

The light-curve of V363 Cas was studied in detail by Hajdu et al. (2009), who identified the true nature of DCEP of this source on the basis of the time-series data collected with the Integral Optical Monitoring Camera (IOMC; Alfonso-Garzón et al. 2012) available at the time (1120 epochs over six years of data). However, because the IOMC has continued to accumulate data, we retrieved all data available so far for V363 Cas and analysed them again. At present, the IOMC dataset comprises 3661 epochs with HJD spanning the range 2452654-2458504 days (i.e. from January 2003 to January 2019). This excellently long time-series was analysed with the Period04 period search software (Lenz \& Breger 2005). We first considered the whole time-series and obtained approximately the same pulsa-

\footnotetext{
2 Unfortunately, we did not observe any $\mathrm{N}$ spectral lines in our spectral range.
}

Table 3. Elemental abundances of V 363 Cas, expressed in terms of solar abundances (Grevesse et al. 2010), for 26 chemical species we measured in our target.

\begin{tabular}{lrrlcr}
\hline \hline $\mathrm{El}$ & \multicolumn{1}{c}{$[\mathrm{El} / \mathrm{H}]$} & $\mathrm{N}$ & $\mathrm{El}$ & {$[\mathrm{El} / \mathrm{H}]$} & $\mathrm{N}$ \\
\hline $\mathrm{Li}$ & $1.76 \pm 0.14$ & 1 & $\mathrm{Mn}$ & $-0.55 \pm 0.12$ & 8 \\
$\mathrm{C}$ & $-0.06 \pm 0.15$ & 4 & $\mathrm{Fe}$ & $-0.30 \pm 0.12$ & 169 \\
$\mathrm{O}$ & $0.00 \pm 0.12$ & 2 & $\mathrm{Ni}$ & $-0.08 \pm 0.11$ & 14 \\
$\mathrm{Na}$ & $-0.11 \pm 0.12$ & 4 & $\mathrm{Cu}$ & $-0.40 \pm 0.10$ & 2 \\
$\mathrm{Mg}$ & $-0.06 \pm 0.12$ & 5 & $\mathrm{Zn}$ & $-0.22 \pm 0.15$ & 3 \\
$\mathrm{Al}$ & $-0.38 \pm 0.15$ & 2 & $\mathrm{Sr}$ & $0.16 \pm 0.10$ & 2 \\
$\mathrm{Si}$ & $-0.08 \pm 0.14$ & 10 & $\mathrm{Y}$ & $0.15 \pm 0.10$ & 3 \\
$\mathrm{~S}$ & $0.06 \pm 0.16$ & 3 & $\mathrm{Zr}$ & $0.08 \pm 0.15$ & 3 \\
$\mathrm{Ca}$ & $0.00 \pm 0.15$ & 4 & $\mathrm{Ba}$ & $0.86 \pm 0.16$ & 5 \\
$\mathrm{Sc}$ & $-0.08 \pm 0.10$ & 3 & $\mathrm{La}$ & $0.33 \pm 0.15$ & 2 \\
$\mathrm{Ti}$ & $-0.06 \pm 0.15$ & 20 & $\mathrm{Ce}$ & $0.05 \pm 0.15$ & 1 \\
$\mathrm{~V}$ & $-0.49 \pm 0.17$ & 5 & $\mathrm{Nd}$ & $0.02 \pm 0.13$ & 5 \\
$\mathrm{Cr}$ & $-0.12 \pm 0.10$ & 17 & $\mathrm{Sm}$ & $0.16 \pm 0.14$ & 4 \\
\hline
\end{tabular}

Notes. Columns labelled with $\mathrm{N}$ represent the number of lines used in the analysis. Results were obtained from the spectrum acquired on November 26, 2019.

tion periods as found by Hajdu et al. (2009), but we also detected highly significant residual peaks around these values in the periodogram. This is a clear indication that the periods are not stable. We then subdivided the IOMC time-series into six chunks that approximately covered the same time span or number of epochs, to be able to derive the periods with sufficient precision and find possible changes. The result of this exercise is presented in Fig. 3 for the P1 pulsation mode because the amplitude of the P2 mode is too small to allow precise results. A clear change in period is seen in the 16 years spanned by the IOMC data, with a quick increase at HJD 2453000 days. To confirm these results, we searched the literature for additional time-series data and found suitable datasets from the HIPPARCos mission (Perryman et al. 1997) and the All-Sky Automated Survey for Supernovae (ASAS-SN, Jayasinghe et al. 2018) surveys. Results from these datasets are also plotted in Fig. 3. Additional insight into the V363 Cas period changes were found in Nowakowski (1988), who analysed historical series of maxima for this star. The authors provided periods that are valid in different epochs (see Fig. 3). We therefore conclude that V363 Cas has a changing period, although at a rather slow rate, because its dominant period increased from $\mathrm{P} 1=0.546517 \pm 0.000013$ days to $\mathrm{P} 1=0.546597 \pm 0.000001$ days overall, that is, 0.00008 days in 100 years, or $\dot{P} / P \sim 1.5 \times 10^{-6}$ year $^{-1}$. However, after HJD 2453000 days, the stellar period increased sharply, corresponding to $\dot{P} / P \sim 1.0 \times 10^{-5}$ year $^{-1}$. This general trend is confirmed by the O-C data-set of V363 Cas in the GEOS RR Lyr database (Le Borgne et al. 2007). 


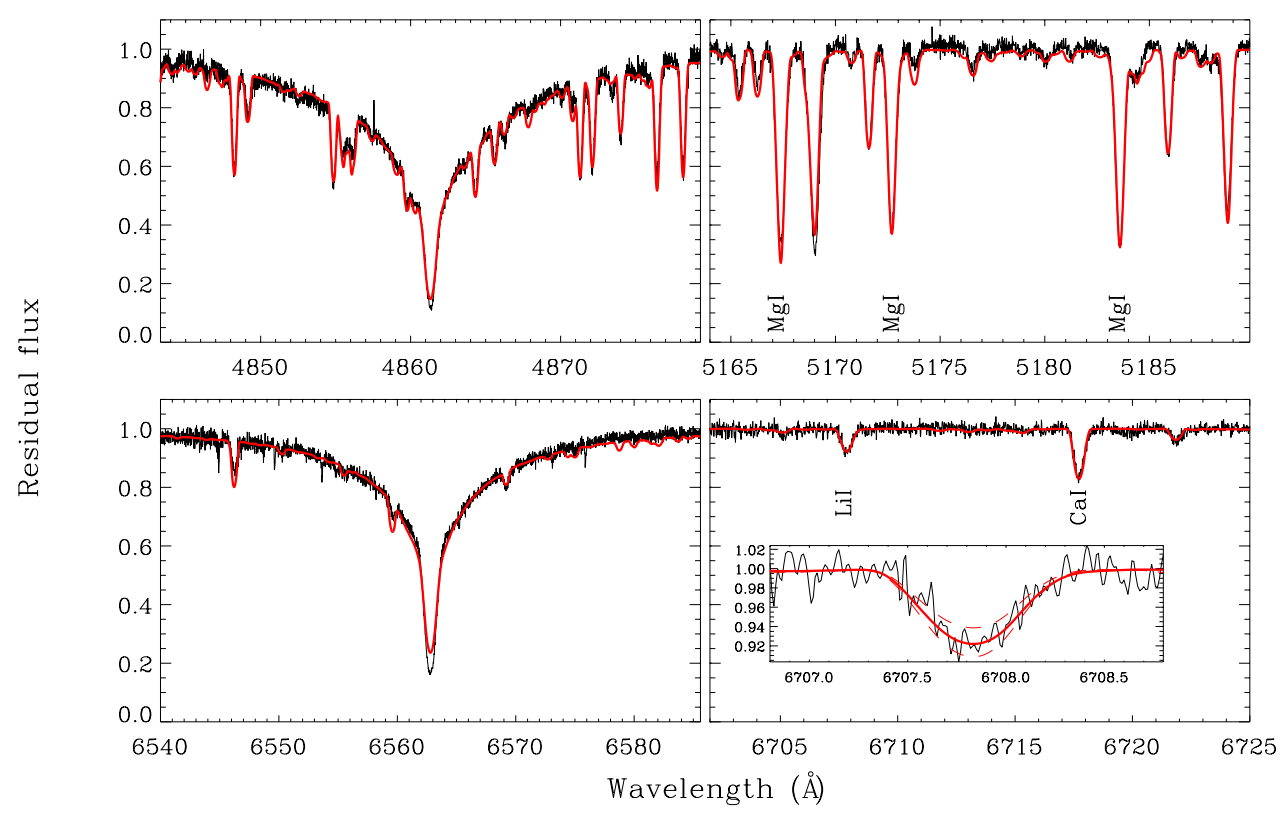

Fig. 2. Comparison between the spectrum of V363 Cas obtained on November 26, 2019 (black line), and the synthetic spectrum (red line) in four main spectral intervals centred on $\mathrm{H} \beta$ (top left panel), the $\mathrm{Mg}$ I triplet at $\lambda \lambda 5167.3216$, 5172.6843, and 5183.6042 $\AA$ (top right panel), $\mathrm{H} \alpha$ (bottom left panel), and the Li I line at $6707.766 \AA$ (bottom right panel), respectively. In the inset, a solid red line shows the fit of the lithium line, and dashed lines represent limits for the experimental error $(\delta= \pm 0.1 \mathrm{dex})$.
To provide updated periods and moments of maximum light for V363 Cas, we performed a period search using only the four most recent chunks of the IOMC time-series dataset because the periods inferred from them are found to be consistent with each other. Results from this procedure are summarised in Table 4, and the folded light curves are shown in Fig. 4.

\section{Discussion and conclusions}

The bolometric luminosity and effective temperature derived in our analysis allows us to place V363 Cas on the HRD. This is shown in Fig. 5, where we also plot the other four MW lithium-rich DCEPs for comparison. We adopted the $T_{\text {eff }}$ values from Kovtyukh et al. (2019) for ASAS J075842-2536.1, ASAS J131714-6605.0, and V371 Per, while for V1033 Cyg we adopted the measure by Udovichenko et al. (2019). The luminosities were estimated by integrating the SEDs, which, as for V363 Cas, were calculated using the VOSA software. Figure 5 also displays the location of the sample of Galactic DCEPs that has recently been analysed by Groenewegen (2020) together with the instability strip for DCEP_F and DCEP_1O of De Somma et al. (2020). We also plot in Fig. 5 the evolutionary tracks by Bressan et al. (2012) in the mass range $M=2.6-$ 5.0 $M_{\odot}$ for the chemical composition $Z=0.01, Y=0.267$ (which is adequate for V363 Cas and V371 Per) and $Z=0.014$, $Y=0.273$ (close to the current solar metal content $Z_{\odot}=0.0152$ and suitable for the remaining stars). V363 Cas is compatible with a mass of $\approx 3.2 M_{\odot}$ and is located at the blue side of the IS, as expected for a multi-mode DCEP pulsating in the 10 and $2 \mathrm{O}$ modes. In addition, the absence of extended blue loops in the tracks encompassing the position of V363 Cas supports our suggestion that V363 Cas is at its first crossing of the IS.

Takeda et al. (2013) carried out a spectroscopic study of 12 evolved DCEPs to investigate evolution-induced mixing in the envelopes of these stars. They derived photospheric abundances of $\mathrm{C}(\approx-0.30 \mathrm{dex}), \mathrm{N}(\approx 0.4-0.5 \mathrm{dex}), \mathrm{O}$ (solar), and $\mathrm{Na}(\approx 0.2$ dex $)$. Moreover, $\mathrm{Li}$ has been found to be very low in DCEPs $(\mathrm{A}(\mathrm{Li})<0.12$ dex Luck \& Lambert 2011). V363 Cas shows almost solar values for both carbon and oxygen (no lines of $\mathrm{N}$ were detected in our spectral range) and a slight underabundance of sodium (but it is still consistent with the solar value

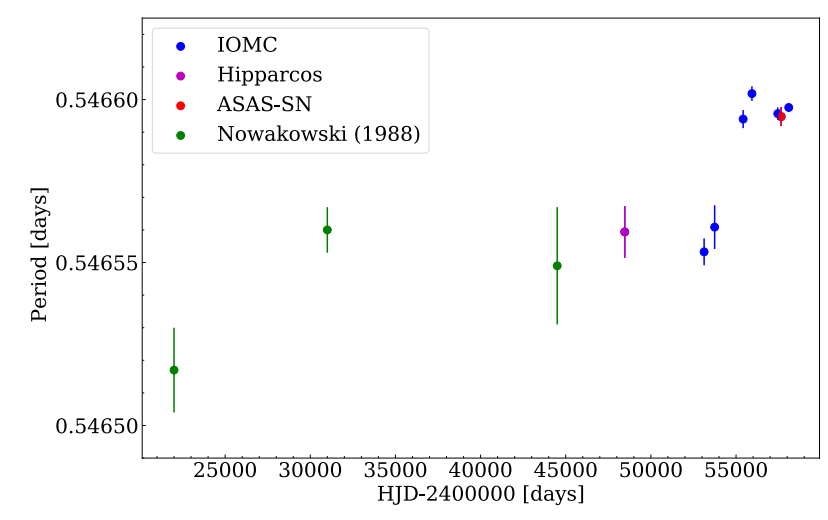

Fig. 3. Period change in the dominant pulsation mode (P1) of V363 Cas. Labels identify the sources of different literature datasets available for this star.

Table 4. Periods, peak-to-peak amplitudes in the $V$ band, and moment of maximum light ( -2400000 days) of V363 Cas.

\begin{tabular}{cccc}
\hline \hline Mode & $\begin{array}{c}\text { Period } \\
\text { days }\end{array}$ & $\begin{array}{c}\text { Amp(V) } \\
\text { mag }\end{array}$ & $\begin{array}{c}\text { HJD }_{\text {Max }} \\
\text { days }\end{array}$ \\
\hline P1 & $0.546597(1)$ & 0.444 & 55201.435 \\
P2 & $0.438083(2)$ & 0.046 & 55201.57 \\
P2/P1 & $0.80133(1)$ & & \\
\hline
\end{tabular}

within the experimental errors). By spectral synthesis of the resonance line at $6707.766 \AA$, we derived $\mathrm{A}(\mathrm{Li})=2.86 \pm 0.1 \mathrm{dex}$. This is consistent with the cosmic abundance and at least $\approx 1.8$ dex higher than the average value found for DCEPs.

In summary, V363 Cas seems to possess all characteristic features of a first-crossing DCEP: it is Li-rich, has solar-like $\mathrm{C}(\mathrm{N}) \mathrm{O}$ abundances (reflecting the photospheric abundance during the MS phase), and shows an increasing period. The multimode nature of V363 Cas is also a common feature among the few known Li-rich MW DCEPs. When V363 Cas is added to the count, four out of five known MW Li-rich DCEPs are found to pulsate in two modes. Additionally, three are 2O/1O pulsators, and only one is a $10 / F$ (V371 Per) pulsator. In this respect, 


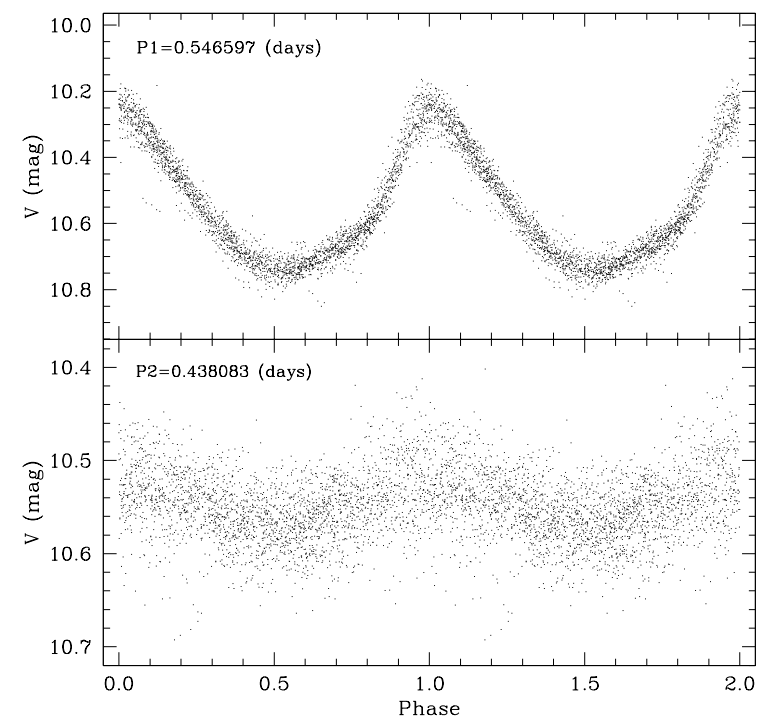

Fig. 4. Light curves of V363 Cas using only the IOMC dataset with HJD in the range of 2455202-2458504 days. The top and bottom panels show the $1 \mathrm{O}(\mathrm{P} 1)$ and $2 \mathrm{O}(\mathrm{P} 2)$ light curves, respectively.

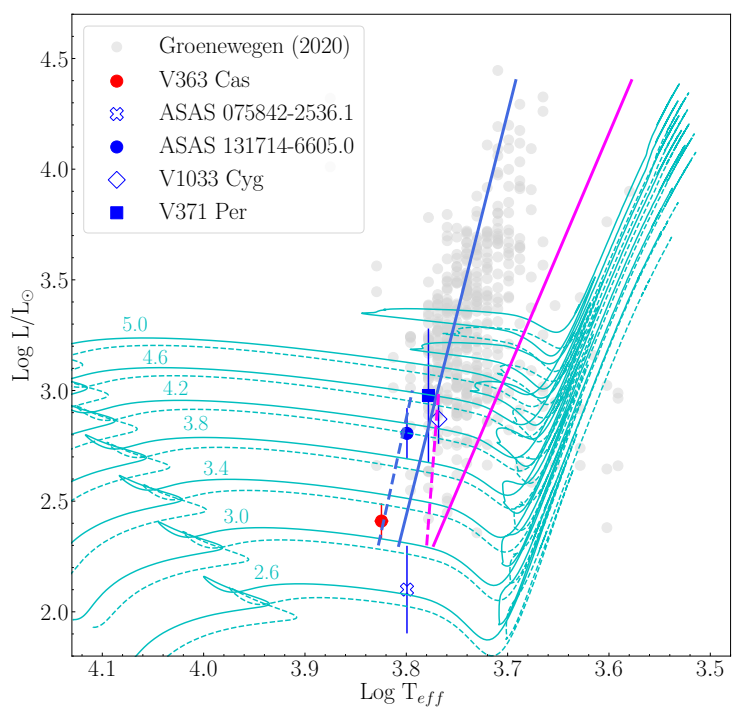

Fig. 5. HR diagram of the known MW Li-rich DCEPs. V363 Cas is shown by the filled red circle. Different symbols (see labels) are used for the other four literature stars. The Li-normal DCEPs by Groenewegen (2020) are shown in light grey as reference. Instability strips for DCEP_F (solid lines) and DCEP_1O (dashed lines) by De Somma et al. (2020) as well as evolutionary tracks by Bressan et al. (2012) for $Z=0.01, Y=0.267$ (solid cyan lines) and $Z=0.014$ $Y=0.273$ (dashed cyan lines) in the mass range 2.6-5.0 $M_{\odot}$ are also over-plotted on the data.

our results support the hypothesis of Kovtyukh et al. (2019) that inefficient mixing in the atmospheres of these DCEPs may have facilitated the preservation of Li. Regarding the position of the MW Li-rich DCEPs on the HRD, ASAS 075852-2536.1 appears to be a low-mass DCEP $\left(\approx 2.6 M_{\odot}\right)$, V1033 Cyg is more massive $\left(\approx 4.4 M_{\odot}\right)$ and alone was shown to have an increasing period, as we now also found for V363 Cas. V371 Per $\left(\approx 4.6 M_{\odot}\right)$ and ASAS 311714-6605.0 $\left(\approx 4.2 M_{\odot}\right)$ are far from the blue loops, according to their tracks. This shows that all these DCEPs are very likely at their first crossing of the IS.
To conclude, the emerging scenario is that a Li-rich DCEP is expected to be at its first crossing (to avoid mixing due to 1DUP) and to have had a low rotation velocity when on the MS (to avoid dilution by rotation mixing, see Brott et al. 2011). New high-resolution observations of MW DCEPs from several groups are ongoing. Therefore more Li-rich DCEPs are likely to be discovered in the near future, which will increase the statistics and help us to unveil the true nature of these stars.

Acknowledgements. This work has made use of data from the European Space Agency (ESA) mission Gaia (https://www.cosmos.esa.int/gaia), processed by the Gaia Data Processing and Analysis Consortium (DPAC, https: //www.cosmos.esa.int/web/gaia/dpac/consortium). Funding for the DPAC has been provided by national institutions, in particular the institutions participating in the Gaia Multilateral Agreement. The Italian participation in DPAC has been supported by Istituto Nazionale di Astrofisica (INAF) and the Agenzia Spaziale Italiana (ASI) through grants I/037/08/0, I/058/10/0, 2014025-R.0, and 2014-025-R.1.2015 to INAF (PI M.G. Lattanzi). We acknowledge partial support from the project "MITiC: MIning The Cosmos Big Data and Innovative Italian Technology for Frontier Astrophysics and Cosmology" (PI B. Garilli). This publication makes use of VOSA, developed under the Spanish Virtual Observatory project supported by the Spanish MINECO through grant AyA2017-84089. VOSA has been partially updated by using funding from the European Union's Horizon 2020 Research and Innovation Programme, under Grant Agreement no 776403 (EXOPLANETS-A). This research has made use of the SIMBAD database, operated at CDS, Strasbourg, France.

\section{References}

Alfonso-Garzón, J., Domingo, A., Mas-Hesse, J. M., et al. 2012, A\&A, 548, A79 Bayo, A., Rodrigo, C., Barrado y Navascués, D., et al. 2008, A\&A, 492, 277B Bressan, A., Marigo, P., Girardi, L., et al. 2012, MNRAS, 427, 127 Brott, I., de Mink, S. E., Cantiello, M., et al. 2011, A\&A, 530, A115 Catanzaro, G., Busá, I., Gangi, M., et al. 2019, MNRAS, 484, 2530 Cyburt, R. H., Fields, B. D., \& Olive, K. A. 2008, J. Cosmol. Astropart. Phys., 11,12

Dambis, A. K., Berdnikov, L. N., Kniazev, A. Y., et al. 2013, MNRAS, 435, 3206 De Somma, G., Marconi, M., Molinaro, R., et al. 2020, ApJS, 247, 30

Fernley, J., \& Barnes, T. G. 1997, A\&AS, 125, 313

Fitzpatrick, E. 1999, PASP, 111, 63

Grevesse, N., Asplund, M., Sauval, A. J., \& Scott, P. 2010, Ap\&SS, 328, 179

Groenewegen, M. A. T. 2018, A\&A, 619, A8

Groenewegen, M. A. T. 2020, A\&A, 635, A33

Hajdu, G., Jurcsik, J., \& Sodor, A. 2009, Inf. Bull. Variable Stars, 5882, 1

Huang, W., Gies, D. R., \& McSwain, M. V. 2010, ApJ, 722, 605

Iben, I. 1967, ARA\&A, 5, 571

Jayasinghe, T., Kochanek, C. S., Stanek, K. Z., et al. 2018, MNRAS, 477, 3145 Kervella, P., Gallenne, A., Evans, N. R., et al. 2019a, A\&A, 623, A117 Kervella, P., Arenou, F., Mignard, F., et al. 2019b, A\&A, 623, A72

Kovtyukh, V. V., \& Gorlova, N. I. 2000, A\&A, 358, 587

Kovtyukh, V., Lemasle, B., Chekhonadskikh, F., et al. 2016, MNRAS, 460, 2077 Kovtyukh, V., Lemasle, B., Kniazev, A., et al. 2019, MNRAS, 488, 3211

Kurucz, R. L. 1993a, A New Opacity-sampling Model Atmosphere Program for Arbitrary Abundances. In: Peculiar Versus Normal Phenomena in A-type and Related Stars, IAU Colloquium 138, eds. M. M. Dworetsky, F. Castelli, \& R. Faraggiana, ASP Conf. Ser., 44, 87

Kurucz, R. L. 1993b, Kurucz CD-ROM 13: ATLAS9 (Cambridge, USA: SAO) Kurucz, R. L., \& Avrett, E. H. 1981, SAO Special Rep., 391

Le Borgne, J. F., Paschke, A., Vandenbroere, J., et al. 2007, A\&A, 476, 307 Lenz, P., \& Breger, M. 2005, Commun. Asteroseismology, 146, 53

Luck, R. E., \& Lambert, D. L. 1992, ApJS, 79, 303

Luck, R. E., \& Lambert, D. L. 2011, AJ, 142, 136

Maeder, A., \& Meynet, G. 2000, ARA\&A, 38, 143

Nowakowski, J. 1988, J. Am. Assoc. Variable star obs., 17, 7

Perryman, M. A. C., Lindegren, L., Kovalevsky, J., et al. 1997, A\&A, 500, 501

Prudil, Z., Dékány, I., Grebel, E. K., et al. 2020, MNRAS, 492, 3408

Romaniello, M., Primas, F., Mottini, M., et al. 2008, A\&A, 488, 731

Takeda, Y., Kang, D.I., Han, I., et al. 2013, MNRAS, 432, 769

Turner, D. G., Abdel-Sabour Abdel-Latif, M., \& Berdnikov, L. N. 2006, PASP, 118,410

Udalski, A., Soszyński, I., Pietrukowicz, P., et al. 2018, Acta Astron., 68, 315

Udovichenko, S. N., Kovtyukh, V. V., \& Keir, L. E. 2019, Odessa Astron. Publ., 32,83 